\title{
How to Choose the Refueling of New Energy Vehicles under Swapping Vs. Charging Mode: From the Consumers' Perspective
}

Jizi Li ( $\boldsymbol{\nabla}$ Jizi.li@ncu.edu.cn )

Nanchang University - Qianhu Campus: Nanchang University

Doudou Wu

Nanchang University - Qianhu Campus: Nanchang University

Chunling Liu

Wuhan Textile University - Yangguang Campus: Wuhan Textile University

Original article

Keywords: New energy vehicles (NEVs), swapping mode (SM), charging mode (CM), pricing, extended warranty services

Posted Date: June 29th, 2021

DOI: https://doi.org/10.21203/rs.3.rs-601348/v1

License: (c) (1) This work is licensed under a Creative Commons Attribution 4.0 International License. Read Full License 
Title:

How to choose the refueling of new energy vehicles under swapping vs. charging mode: from the consumers' perspective

Jizi Li ${ }^{*}$, Doudou Wua, Chunling Liu ${ }^{\mathrm{b}}$

a School of Management, Nanchang University, Nanchang, 330031, China

${ }^{\mathrm{b}}$ Research Center of Supply Chain System, Wuhan Textile University, Wuhan, 43007, China

E-mail address: jizi.li@ncu.edu.cn (J. Li), wudoudou@email.ncu.edu.cn (D. Wu), liuring@wtu.edu.cn (C. Liu)

* Corresponding author: jizi.li@ncu.edu.cn (J. Li) 


\title{
How to choose the refueling of new energy vehicles under swapping vs. charging mode: from the consumers' perspective
}

\begin{abstract}
Background: Battery charging mode is a prevalent method of transshipping power to new energy vehicles. Unfortunately, due to the limited capacity of batteries, typical new energy vehicles can only travel for approximate 350 miles on a single charge and require hours to be recharged. Battery swapping mode, as a novel alternative, can offer ideal solutions by depleted batteries being exchanged for recharged ones at swapping stations in the middle of long trips, thus will inevitably influence potential consumers' purchase behaviors accordingly.
\end{abstract}

Methods: To examine the impact of swapping mode and charging mode on consumers' purchase intention, this paper examines a duopolistic market consisting of two new energy vehicle makers (i.e., new energy vehicle maker with swapping mode and one with charging mode), who adopting swapping mode and charging mode to service consumers, respectively. Considering swapping mode characterized with low initial investment and ease of use for consumers, the new energy vehicle makers with charging mode, capitalize on battery extended warranty service in response to rivals' utilization of swapping mode, thereby non-cooperative game models are formulated, in which government subsidies are taken into account, both the optimal production decision for the new energy vehicle maker with swapping mode maker and one with charging mode are analyzed under three scenarios: without extended warranty service, with extended warranty service, and with extended warranty service and subsidy.

Results: The results show that two makers' market dominance relies on the ratio of the swapping station's convenience to the extended warranty service and the valuation incremental rate. Additionally, we also find that the government subsidy can dramatically improve the performance of the new energy vehicle maker with swapping mode at the initial stage, but if the subsidy is insufficient size at the sequent stage, this will lead to policy failure and inefficiency in propelling the diffusion of swapping mode.

Conclusions: The emerging swapping mode technology is a promising innovative one in boosting new energy vehicle diffusion, which will supplement to charging mode, thus reduce climate change dramatically

Keywords: New energy vehicles (NEVs); swapping mode (SM); charging mode (CM); pricing; extended warranty services

\section{Introduction}

Compared with traditional fuel vehicles (FVs), new energy vehicles (NEVs) have more potential benefits, and are considered as the future alternative to alleviate global greenhouse gas emissions (Šare, et al. 2015; Li et al, 2020; Liu et al, 2020). However, there exist a couple of critical obstacles to overcome before NEVs will be used on a mass scale. Perhaps the most significant challenge stems from the nature of recharging. Whereas fuel vehicles (FVs) can be easily refueled at accessible gas stations in a matter of minutes, NEVs require specific equipment and a significant amount of time (hours) to be recharged (Liu et al, 2016). The shorter cruise range of NEVs than that of fuel vehicles (FVs), which necessitates more frequent recharges than gasoline refuels, only makes this problem worse. Whereas it is normal to park the NEVs at a workplace or home for a few hours or overnight, it is impractical to station the car for a recharge in the middle of a long trip (Li et al, 2019; Yao \& Luo, 2018). 
Battery swapping mode (SM) can effectively solve the aforementioned problem (Schiffer and Walther, 2017; Moghaddam et al, 2018). In this mode, NEV makers (or battery swapping service providers) deploy the network of swapping stations where NEV' users may exchange their depleted batteries for full ones in the middle of long trips. The depleted batteries are then recharged at the stations and later swapped for depleted batteries on other arriving NEVs. This solution allows NEVs to be effectively "refueled" in only one to two minutes instead of hours( Moghaddam et al, 2018). Different from the conventional charging mode (CM), SM is somewhat analogous to cell phone service providers. It builds and operates the infrastructure system (i.e., battery-swapping stations) and provides service ("refueling" NEVs). Because batteries can be swapped, they are not owned by the NEV users themselves but rather leased to them based on some service contracts. Users will be charged based on usage (i.e., miles driven), much like cell-phone users being billed on talk time.

This arrangement poses several potential benefits. It decouples ownerships of the battery and the NEV, two items with different life cycles, and makes it very easy to take advantage of future improvements in battery technology by regular battery replacements, compared with other solutions that bundle the NEV and battery as one single unit (Hof et al., 2017). Most importantly, the significantly lower upfront cost for the user to purchase a new NEV (the battery typically costs around $\$ 10,000$ ) can help boost adoption rates. Second, swapping mode can effectively lower the risk of battery's damage in the fast-direct charging mode, it estimates that the frequently adopting fast direct charging for NEVs shorten the life of battery by approximate $20 \%$ due to the strong electric flows. Third, swapping mode can be utilized to recharge at night rather than at daytime, which dramatically reduce electricity fees, and balance the power load and decrease the difference of the peak and valley load for the power grid side. Besides, swapping mode could be helpful in implementing the life-cycle management of battery. The average battery life span is approximately 8 years due to degradation in capacity. Unlike fuel vehicles, NEVs cannot use their power batteries until they reach the end of their life-cycle. Instead, the power batteries have to be removed when their capacities fall to around $70-80 \%$ due to performance and safety concerns (McIntire-Strasburg, 2015), and yet can reuse in other fields including energy storage for wind and solar power, thus in turn cut down operational costs.

Therefore, the government and carmakers are attracted to boost SM with an aim to penetration of NEVs. For example, China, as the largest NEV's production and sales country, has specifically announced battery swapping network as a core component of the new infrastructure strategy' for NEV charging and will offer fiscal supports (China Daily, 2020), and an ambitious battery swapping-station deployment plan is under way in major cities (China Daily, 2019). In addition, some major leading China's NEV-SM makers like BAIC and Nio Motor are promoting battery swapping mode in Taxi business and individual consumers, respectively. BAIC has built and opened 187 swapping stations in 19 cities in China including Beijing, Xiamen, Lanzhou and Guangzhou, servicing 18000 swapping taxis with 4.8 million times, and the total travel mileage has reached 690 million $\mathrm{km}$. Particularly, Beijing has more than 100 BAIC swapping stations, whose network can meet the service radius of $2.53 \mathrm{~km}$ in urban area, that is to say, one station can be found within $2.53 \mathrm{~km}$ distance, thus make drivers perceive the convenience similar to that of gas stations. In addition, according to the official data of Nio Motor, more than 140 swapping stations are opened nationwide, offering more than 0.8 million times of swapping service. Each station can operate for 96 vehicles a day at most. From January to July 2020, the cumulative sales volume of Nio Motor is 14,321 units, accounting for 3.59\% of China's total NEV sales market (398,000 units), which reveal the positive trend in NEV-swapping mode development (China Daily, 2020). 
Although SM displays remarkable merits, some problems, particularly the insufficient number of swapping stations, hamper the shape of an efficient supply network to service consumers, meanwhile, $\mathrm{CM}$ still dominates in NEV market, NEV-CM makers (i.e., new energy vehicle maker with charging mode) usually implement various strategies including extended warranty service to counteract NEV-SM makers (i.e., new energy vehicle maker with swapping mode). Spurred by these practices, we address three questions in this paper: (1) What is the condition in which both NEV-SM makers and NEV-CM makers obtain the equilibrium? (2) In a duopolistic market, whether the NEV-SM maker possesses competitive advantage over its rivals considering battery extended warranty service? (3) Which demand differences occur between the NEV-SM makers and the NEV-CM maker under government subsidy? How government subsidy affects consumer surplus and social welfare at different development stage swapping network?

To handle the aforementioned problems, we present our theoretical models under base model without and with extended warranty service, and then extend the model incorporating government's subsidies. The main academic contributions of this paper are threefold, (1) different from prior work, we examine the impact of battery swapping mode (SM) in a competitive environment, where two NEV makers adopt different types of recharge (i.e. swapping mode and charging mode), rather than study it alone; (2) we employ the critical factor of battery extended warranty service to investigate the optimal pricing for both NEV makers, which seldom address before; (3) piecewise government subsidies are taken into account to analyze the difference in optimal outcomes.

The remainder of this paper proceeds as follows. Section 2 reviews the most relevant literature. In Section 3 we present our theoretical models and the corresponding results. Subsequently, numerical study and sensitivity analysis are conducted in Section 4 . Section 5 provides concluding remarks and future research direction. All proofs are offered in Appendix A.

\section{Literature review}

This paper investigates the optimal decision for new energy vehicles with battery swapping/charging mode in the context of extended warranty service. The main relevant studies include two domains, namely, battery swapping/charging mode and extended warranty service.

\subsection{Research of battery swapping/charging mode for NEVs}

The literature regarding NEV swapping mode mainly address the locations of swapping stations, comparison of swapping mode and direct charging mode for an operator under various scenarios. Yang and Sun (2015) investigate electric vehicles battery swap stations location, and the routing plan of a fleet of electric vehicles simultaneously. Schiffer and Walther (2017) relax the constraint of battery driving range limitation, to examine the routing of electric vehicles and sitting decisions for charging stations in order to support strategic decisions of logistics fleet operators. Results show that the shorter overall distances can be achieved if simultaneous sitting as well as extended recharging options are allowed. Hof et al. (2017) further examine the battery swap station location-routing problem with taking capacitated electric vehicles into account. More specifically, Wu et al. (2018) emphasize three factors, namely, the number of batteries from stock to serve incoming EVs, potential charging damage, and electricity cost, to study the optimized charging schedule for swapped electric vehicle batteries, and compare the performance between two different algorithms.

Unlike the aforementioned work only focusing on battery swapping mode, Liu et al. (2016) establish the dispatching models of micro-grid with EVs in different energy supply modes, namely, plug-in charging mode and battery swapping mode. The results demonstrate the micro-grid with EVs in battery 
swapping mode is more economic compared with the EV plug-in charging mode, and the effectiveness of the dispatching model. Furthermore, Moghaddam et al. (2018) model a multi-objective optimization problem (i.e., the minimum charging time, travel time, and charging cost) of a EV network that offers multiple charging options, including normal charging, fast charging, and battery swapping facilities, and design a meta-heuristic solution in the form of an ant colony optimization. Simulation results show that the proposed solution significantly reduces waiting time and charging cost.

In particular, for various operators, Zhang et al. (2016) examine and compare a microgrid operator performance using the battery swapping mode and direct charging mode under the wind power and photovoltaic. The results showing that the battery swapping mode is better than the direct charging mode for using battery discharging energy when energy price is high, and charging when energy price is low, thus improving the operational economy of the micro-grid. In parallel, $\mathrm{Wu}$ (2019) examines a EV-sharing operator under battery-charging technology (battery swapping mode and direct charging mode) and insurance contract (i.e., Fixed-premium insurance and per-hour-premium insurance), developed a stylized model with which to analyze the impact of different charging technologies, insurance contracts and other related factors on the EV-sharing operator's profit.

Different from extant literature mainly addressing an operator's choice between swapping mode and direct charging mode, we examine the best choice of two NEV-makers adopting swapping mode and direct charging mode, respectively and competing in the same NEV market.

\subsection{Research of extended warranty service}

For durable consumer products, after-sale services play an important role in customers' purchase decisions (Kurata and Nam, 2010). The basic warranty as an after-sale service is available to product owners via offering a free repair and replacement, except for basic warranty, extended warranty service has become popular, Zheng et al. (2018) examine a extended warranty under which an additional trade-in service is provided during the warranty coverage, and find that the new extended warranty does not be offered at a higher price than basic warranty, but when the handling cost for used products is relatively low, the extended warranty will outperform basic warranty. Tong et al. (2014) consider a base warranty consisting of two-dimensional region of age and usage, and analyze a designing and pricing model of two-dimensional extended warranty contracts. Unlike the one-price-for-all extended warranties, Gallego et al. (2015) differentiate customers according to their risk attitudes and usage rates, and design residual value warranties, find the residual value warranties can better price discriminate customers than traditional warranties with contingent contracts. More specifically, Nie and Deng(2014) develop three models, namely, no extended warranty services, manufacturers provide extended warranty services and the third-party provide extended warranty services, and take the quality as an endogenous variable.

Extended warranty services also influences its partners, Yi and Yao (2014) research the influence of network externalities on extended warranty in a two-stage supply chain model, the findings reveal that the supply chain with the retailer and supplier could be coordinated and obtained Pareto improvement by improved revenue-sharing extended warranty contract. Meanwhile, Zhao et al. (2019) examines service supply chain optimal pricing in the context of vertical competition with two extended warranty cases: one considering the retailer's fairness concerns and one without considering the retailer's fairness concerns, and finds two parties of the supply chain employ differential pricing strategies for extended warranties when the retailer has fairness concerns. He et al. (2018) also build three game-theoretic models to study how to design service strategies of extended warranty in a dual supply channel by 
taking into account customer channel preferences. Furthermore, Ma et al. (2015) further considers two supply chains engaged in price competition with the manufacturers provide pay-needed extended warranty services, and investigate the equilibrium for channel structure choices with different lengths of extended warranty period and competition status.

The second stream of research studies extended warranty strategies in various conditions and demonstrate that warranty strategy are important to firms. In contrast, our paper investigates the interaction between extended warranty and swapping network deployment in a swapping/charging competitive context, where two different types of NEV makers sell their products to heterogeneous consumers, which seldom study before.

\section{Theoretical models}

We study a duopolistic market where exist two NEV makers, namely a NEV-SM maker and a NEV-CM maker, the NEV-SM maker produces and sells NEVs with the adoption of battery swapping mode, whereas the NEV-CM counterpart makes NEVs with charging mode. Two NEV products are sold to consumers in price $p_{1}$ and $p_{2}$, respectively. Consider that the NEV-CM maker who bundling the NEV and battery as a whole to sell consumers, and a consumer preferring the NEV-SM products merely buys the NEV and rents power battery, thus it is reasonable to let $p_{1}<p_{2}$.

With two different ways of transshipping power to NEVs (i.e., SM and CM), heterogeneous consumers' purchase behaviors are independent and separated. We assume that consumers are heterogeneous in product valuation. Similar to the previous study, the product valuation is represented by $v$, and $v$ is uniformly distributed over $[0,1]$. It means that when consumers buy vehicles, they consider various factors such as price, appearance, convenience, warranty period and among others. Compared to $\mathrm{CM}$, SM can bring convenience to consumers in terms of initial cost and recharging time, in parallel, consumers will also consider whether the number of battery swapping stations $m$ is sufficient as to meet their travel requirements after buying NEVs with swapping mode. In reality, it is true that the current swapping network is at an infant stage, which hinders the NEV-SM products to be widely used to some degrees. Main notations used in this paper are summarized in Appendix (Table 1).

Therefore, one perspective consumer may have three alternatives to choose: (1) purchase a NEV with SM, (2) purchase a NEV with CM, and (3) buy nothing. Referencing the literature (Michael \& Rosen, 1978), the three utilities of consumers are formulated as follows:

$$
\begin{aligned}
u_{1} & =v-p_{1}+\delta m \\
u_{2} & =v(1+\lambda)-p_{2}
\end{aligned}
$$

Eq.(1) indicates that consumers' utility function of the NEV-SM is related with the valuation $v$, number of battery swapping station $m$, and price $p_{1}, \delta$ is denoted by the battery swapping stations convenience coefficient. While Eq.(2) shows that of the NEV-CM depends on $v, p_{2}$, and $\lambda$ the valuation incremental rate of CM relative to SM. It is easy to know that consumers' utility $u_{1}>0$ and $u_{2}>0$, and when $\Delta u=u_{1}-u_{2}>0$, consumers are willing to purchase NEV-SM rather than NEV-CM; otherwise, when $\Delta u=u_{1}-u_{2}<0$, consumers prefer NEV-CM instead of NEV-SM, hence we have

$$
\Delta u=p_{2}-\lambda v-p_{1}+\delta m
$$

Due to that consumers are heterogeneous in product valuation $v$ uniformly distributed over $[0,1]$. Eq.(4) yields the decoupling point between purchasing a NEV-SM and purchasing a NEV-CM. We can denote this point by $v_{0}$, which is represented by $v_{0}=\left(p_{2}-p_{1}+\delta m\right) / \lambda$. Therefore, it is easy to derive demand function regarding NEV-SM and NEV-CM, respectively. 


\subsection{Base Model}

$$
\begin{aligned}
D_{1} & =\int_{0}^{\left(p_{2}-p_{1}+\delta m\right) / \lambda} d v=\left(p_{2}-p_{1}+\delta m\right) / \lambda \\
D_{2} & =\int_{\left(p_{2}-p_{1}+\delta m\right) / \lambda}^{1} d v=\left(\lambda-p_{2}+p_{1}-\delta m\right) / \lambda
\end{aligned}
$$

In this subsection, we take into account the scenario where the NEV-CM maker dose not leverage extended warranty service to compete with the NEV-SM maker in NEV market.

Consider that building swapping infrastructure need the NEV-SM maker investing fix costs. Obviously, the fixed cost is closely related with the number of battery swapping station $m$, based on the literature (Lau et al., 2012), the battery swapping station costs can be presented as a quadratic form $\alpha \mathrm{m}^{2} / 2, \alpha$ is the cost coefficient of battery swapping station. Additionally, supposed that two firms produce NEVs with the same production cost structure $c_{1}=c_{2}=c$.

The profits of the NEV-SM maker and the NEV-CM maker are expressed as follows:

$$
\begin{aligned}
& \pi_{1}\left(p_{1}, m\right)=D_{1}\left(p_{1}-c_{1}\right)-\frac{1}{2} \alpha m^{2} \\
& \pi_{2}\left(p_{2}\right)=D_{2}\left(p_{2}-c_{2}\right)
\end{aligned}
$$

Note that, the first term of formula (6) represents the sales revenue from NEV-SM, and the second term denotes the fixed cost of building battery swapping stations. Formula (7) represents the profit from NEV-CM. Putting Equations (4) and (5) into (6) and (7), we obtain

$$
\begin{aligned}
\pi_{1}\left(p_{1}, m\right) & =\left(p_{1}-c\right)\left(p_{2}-p_{1}+\delta m\right) / \lambda-\frac{1}{2} \alpha m^{2} \\
\pi_{2}\left(p_{2}\right) & =\left(p_{2}-c\right)\left(\lambda-p_{2}+p_{1}-\delta m\right) / \lambda
\end{aligned}
$$

The NEV-SM maker and the NEV-CM maker determine their own optimal selling prices with an aim to maximize his own profit, using the backward induction to solve formula (9) and (10).

Proposition 1. Under the swapping mode Vs. charging mode, if $\lambda>\delta^{2} / 2 \alpha$ holds on, the equilibrium outcomes are $p_{1}{ }^{*}=\frac{3 \alpha c \lambda+\alpha \lambda^{2}-c \delta^{2}}{3 \alpha \lambda-\delta^{2}}, m^{*}=\frac{\lambda \delta}{3 \alpha \lambda-\delta^{2}}, p_{2}^{*}=\frac{3 \alpha c \lambda+2 \alpha \lambda^{2}-c \delta^{2}-\lambda \delta^{2}}{3 \alpha \lambda-\delta^{2}}$,

$$
D_{1}^{*}=\frac{\alpha \lambda}{3 \alpha \lambda-\delta^{2}}, D_{2}^{*}=\frac{2 \alpha \lambda-\delta^{2}}{3 \alpha \lambda-\delta^{2}} .
$$

Lemma 1. Only if inequalities $\lambda>\delta^{2} / \alpha$ holds, the optimal solutions in the base model are achieved; whereas if $\delta^{2} / \alpha>\lambda>\delta^{2} / 2 \alpha$ satisfies, although the equilibrium outcomes can be obtained, the optimal solutions do not exist.

Lemma 1 shows the current situation where the CM mode dominates in NEV market and yet the SM mode is still at an infant phase, only when $\lambda$ exceeds a certain threshold value (i. e.,$\lambda>\delta^{2} / \alpha$ ), such that consumers perceive the price of SM-NEVs is less than that of CM-NEVs (i.e., $p_{1}<p_{2}$ ), the NEV-SM can attract consumers to purchase, which is consistent with the reality. In this regard, both the NEV-CM maker and the NEV-SM maker obtain optimal profits under swapping mode Vs. charging mode.

Theorem 1. Only if $\lambda>\delta^{2} / \alpha$ satisfies, the NEV-SM maker can keep its price lower than that of its rival NEV-CM maker, but the NEV-CM maker always outperforms the NEV-SM maker in term of market demand and profit, i.e., $D_{1}<D_{2}, \pi_{1}<\pi_{2}$.

Theorem1 indicates that, at the initial development stage of NEV-SM, the NEV-SM maker adopts the low-price strategy, with aims to tap into the market and attract potential consumers, yet this move is not a long-term solution due to that the NEV-CM maker invariably occupies more market shares and profits in the NEV market. With the decrease of $\lambda$, i.e., the valuation change rate of CM relative to SM, the NEV-SM maker will get better off, because consumers gradually realize the NEV-SM's advantages over that of the NEV-CM, it is why NIO Motors, one of leading NEV-SM makers, is attracting more and 
more customers to choose the SM mode in China.

Corollary 1. Under the swapping mode Vs. charging mode, when $\lambda \in\left(\delta^{2} / \alpha, \infty\right), \partial \pi_{1} / \partial \alpha<0, \partial \pi_{1} / \partial \delta>0$.

Corollary 1 shows that, when $\lambda \in\left(\delta^{2} / \alpha, \infty\right), \pi_{1}$ decreases in $\alpha$, but increases with $\delta$. The result is intuitive, because the parameter of $\alpha$ represents the construction cost per swapping station, the increase of $\alpha$ will inevitably lead to the NEV-SM maker's profit reduction. Likewise, the increase of $\delta$ means consumers' perception of usage of NEV-SM products become more appealing, thus drive people to purchase it, which in turn increase in the NEV- SM maker's profitability. It reminds managers to realize the important role of the swapping network convenience in practice, in other words, the NEV-SM maker not only endeavor to expand the network infrastructure, but emphasize the location of swapping station as well.

\subsection{Model with extended warranty service}

Under this model, due to that NEV-SM maker leases batteries to consumers rather than sell them bundled with vehicles, thus significantly lower consumers' purchase costs and the subsequent battery maintenance fees. To counteract its rival's merit, the NEV-CM makers are consolidating their dominant market position by providing consumers with the after-sales service for batteries, namely extended warranty service, in an attempt to overcoming its short broad. For instance, WeltMeister, as another leading China's NEV-CM maker, launched the nationwide battery extended warranty service in 2018, thus lead to peers' follow-up including BYD and Xpeng Motors. With the extended warranty service, we define $T$ as the duration of extended warranty service, $k$ represents the extended warranty service coefficient. Similar to the base model, the consumer utility regarding NEV-CM in setting of extended warranty service policy is formulated as:

$$
\widetilde{u_{2}}=(1+\lambda) v-\widetilde{p_{2}}+k T
$$

While the consumer utility regarding NEV-SM remains unchanged, As the same reasoning as before, we derive out the demand functions of NEV-SM and NEV-CM, respectively.

$$
\begin{gathered}
\widetilde{D_{1}}=\frac{1}{\lambda}\left(\widetilde{p_{2}}-\widetilde{p_{1}}+\delta \widetilde{m}-k T\right) \\
\widetilde{D_{2}}=\frac{1}{\lambda}\left(\lambda+\widetilde{p_{1}}-\widetilde{p_{2}}-\delta \widetilde{m}+k T\right)
\end{gathered}
$$

Thus the profit functions of NEV-SM and NEV-CM under the extended warranty service are expressed as below,

$$
\begin{gathered}
\widetilde{\pi_{1}}\left(\widetilde{p_{1}}, \widetilde{m_{1}}\right)=\widetilde{D_{1}}\left(\widetilde{p_{1}}-c_{1}\right)-\frac{1}{2} \alpha \widetilde{m}^{2} \\
\widetilde{\pi_{2}}\left(\widetilde{p_{2}}, T\right)=\widetilde{D_{2}}\left(\widetilde{p_{2}}-c_{2}\right)-\beta T^{2} / 2
\end{gathered}
$$

Obviously, the first term of formula (12) entails the sales revenue from NEV-CM, and the second term denotes the NEV-CM maker's incurring the cost of extended warranty service $\beta T^{2} / 2$, where $\beta$ is the cost coefficient of extended warranty service. We can obtain the optimal decisions and two firms' profit by finding the first order derivative of formula (14) and (15).

Proposition 2. Under the extended warranty service, if $\alpha k^{2}>\beta \delta^{2}, \lambda>k^{2} / 2 \beta$ or $\alpha k^{2}<\beta \delta^{2}, \lambda>\delta^{2} / 2 \alpha$ hold on, the equilibrium outcomes are

$$
\begin{aligned}
& T^{*}=\frac{-k\left(2 \alpha \lambda-\delta^{2}\right)}{\alpha k^{2}+\beta \delta^{2}-3 \alpha \beta \lambda^{\prime}} \quad \widetilde{m}^{*}=\frac{\delta\left(k^{2}-\beta \lambda\right)}{\alpha k^{2}+\beta \delta^{2}-3 \alpha \beta \lambda^{\prime}} \widetilde{p_{1}^{*}}=\frac{\alpha\left[-\beta\left(3 c \lambda+\lambda^{2}\right)+k^{2}(c+\lambda)\right]+c \beta \delta^{2}}{\alpha k^{2}+\beta \delta^{2}-3 \alpha \beta \lambda}, \\
& \widetilde{p_{2}^{*}}=\frac{\beta\left[-\alpha\left(3 c \lambda+2 \lambda^{2}\right)+\delta^{2}(c+\lambda)\right]+c \alpha k^{2}}{\alpha k^{2}+\beta \delta^{2}-3 \alpha \beta \lambda}, \widetilde{D_{1}^{*}}=\frac{\alpha\left(k^{2}-\beta \lambda\right)}{\alpha k^{2}+\beta \delta^{2}-3 \alpha \beta \lambda}, \quad \widetilde{D_{2}^{*}}=\frac{-\beta\left(2 \alpha \lambda-\delta^{2}\right)}{\alpha k^{2}+\beta \delta^{2}-3 \alpha \beta \lambda}
\end{aligned}
$$

Theorem 2. In the setting of the extended warranty service, 
(i) When $0<\frac{\delta}{k}<(2 \alpha / \beta)^{\frac{1}{2}}, \lambda>k^{2} / \beta$ hold on, the NEV-SM maker' profit takes advantage over the NEV-CM maker, whereas the NEV-SM maker covers less market shares than its rival. (i.e., $\widetilde{D_{1}^{*}}<\widetilde{D_{2}^{*}}, \widetilde{\pi_{1}^{*}}>\widetilde{\pi_{2}}{ }^{*}$ );

(ii) When $\frac{\delta}{k}>(2 \alpha / \beta)^{1}, \lambda>\left(\beta \delta^{2}-\alpha k^{2}\right) / 2 \alpha \beta$ hold on, the NEV-CM maker is more competitive than the NEV-SM maker (i.e., $\widetilde{D_{1}^{*}}<\widetilde{D_{2}^{*}}, \widetilde{\pi_{1}}{ }^{*}<\widetilde{\pi_{2}}{ }^{*}$.

Theorem 2 displays that the consumers' choice of two modes relies on two critical factors, namely, $\lambda$ and $\frac{\delta}{k^{\prime}}$ where $\frac{\delta}{k}$ denotes the ratio of the swapping station's convenience coefficient to the extended warranty service coefficient. When the coefficient ratio does not surpass a specific threshold value $(2 \alpha / \beta)^{1}$, and the valuation incremental rate of CM relative to SM is at high level, although the NEV-SM maker's demand is less than that of its competitor, its profit still surpasses the NEV-CM maker's. On the other hand, when the valuation incremental rate of $\mathrm{CM}$ relative to SM remain a relatively high level, but the coefficient ratio is above a specific threshold value $(2 \alpha / \beta)^{2}$, the NEV-CM maker dominates market in terms of demand and profit. The results imply that regardless of $0<\frac{\delta}{k}<(2 \alpha / \beta)^{\frac{1}{2}}$ or $\frac{\delta}{k}>(2 \alpha / \beta)^{2}$, the NEV-CM is more appealing to consumers from the demand perspective. Additionally, $0<\frac{\delta}{k}<(2 \alpha / \beta)^{\frac{1}{2}}$, the inequality $\lambda>k^{2} / \beta$ ensures that the price of NEV-SM product is less than that NEV-CM one, yet the NEV-SM maker's payoff is larger than its rival due to that the cost of extended warranty service. In contrast, given $\frac{\delta}{k}>(2 \alpha / \beta)^{2}$, the inequality $\lambda>\left(\beta \delta^{2}-\alpha k^{2}\right) / 2 \alpha \beta$ guarantees the price of NEV-SM product is less than that NEV-CM one, the NEV-CM maker's profit is larger than its rival, because of the higher value of $\lambda$. It implies that even if the extended warranty service temporarily has a negative impact on the NEV-CM maker's performance due to initial investment, but its market dominance indeed is enhanced further.

Corollary 2. In the setting of the extended warranty service,

(i) When $2 \alpha k^{2}>\beta \delta^{2}, \lambda>k^{2} / \beta$, both $\widetilde{m}$ and $T$ increase with $\delta$;

(ii) When $2 \alpha k^{2}<\beta \delta^{2}, \lambda>\left(\beta \delta^{2}-\alpha k^{2}\right) / 2 \alpha \beta$, $\widetilde{m}$ increases with $\delta$; while $T$ decreases with $\delta$.

Corollary 2 exhibits the monotonicity of $\delta$ in the setting of the extended warranty service. The outcomes indicate that, regardless of what conditions are, the increase of number of swapping station $(\widetilde{m})$ will enhance the perceived convenience of swapping mode $(\delta)$, which is consistent with the reality, but for the extended-warranty-service duration (T), the impact of $\delta$ shows the differences, when $2 \alpha k^{2}>$ $\beta \delta^{2}, \lambda>k^{2} / \beta, T$ has a positively influence on $\delta$, it means that the NEV-SM maker improves the convenience of swapping mode $(\delta)$, which triggers its rival to expand the extended-warranty-service duration $(T)$ for batteries with an aim to avoiding the loss of customers. However, when $2 \alpha k^{2}<\beta \delta^{2}$, $\lambda>\left(\beta \delta^{2}-\alpha k^{2}\right) / 2$, the increase of $\delta$ will make the NEV-CM maker to shorten the extended-warranty-service duration $(T)$, the rationale of such result is that, from Theorem 2 know when $2 \alpha k^{2}>\beta \delta^{2}, \quad \lambda>k^{2} / \beta$, the NEV-CM maker's profit is lower than its competitors, thus leading to his aggressive response to the NEV-SM's move, whereas when $2 \alpha k^{2}<\beta \delta^{2}, \lambda>\left(\beta \delta^{2}-\alpha k^{2}\right) / 2 \alpha \beta$, the NEV-CM maker dominate the NEV market in term of demand and profit, the shortening of the extended-warranty-service duration $(T)$ may suitable for more perspective consumers' needs.

\section{The impact of subsidy policy}

From the above analysis, to effectively compete with the NEV-CM maker, the NEV-SM maker should improve its swapping network for enhancing the replacement battery convenience, hence requiring an increase in the number of swapping stations, which in turn relies on the investment from two sources including government subsidy. In this section, we further analyze the impact of government 
subsidy policy on two different mode selection. Although governments gradually cut down the amounts of NEV subsidies until completely abolish monetary aids, the subsidies for the construction of swapping stations still remain unchanged, because the NEV-SM makers are unaffordable to invest in swapping infrastructure deployment alone at the initial stage of promoting SM. To speed up the sitting-and-sizing process of swapping stations, governments usually provide financial subsidies to encourage the NEV-SM makers to perfect their swapping network. In this regard, we assume subsidies are allocated to the NEV-SM maker, rather than to the NEV-CM maker.

The common practice is that the subsidy size relies on the number of power swapping stations built. In addition, when the swapping network starts to take shape, the subsidy amount will somewhat reduce accordingly. Thus, government's subsidy represents a piecewise function as below,

$$
f(s)=\left\{\begin{array}{lc}
a m_{s}, & 0<m_{s} \leq m_{1} \\
a m_{1}+b\left(m_{s}-m_{1}\right), & m_{1}<m_{s} \leq m_{2}
\end{array}\right.
$$

Where $m_{1}$ represents the minimal number of swapping stations below which point, i.e., $m_{s} \in$ $\left(0, m_{1}\right)$, the NEV-SM maker can obtain $a$ subsidies per station, while $m_{2}$ stands forthe maximal number of swapping stations above which point the subsidy size per station will decrease to $b$ from $a$ $(0<b<a)$,i.e., $m_{s} \in\left(m_{1}, m_{2}\right)$. To this end, $(s)=a m_{s}, m_{s} \in\left(0, m_{1}\right)$, it means the swapping network construction is at the preliminary stage $(\mathrm{I})$, while when $f(s)=a m_{1}+b\left(m_{s}-m_{1}\right), m_{s} \in\left(m_{1}, m_{2}\right)$, it signals that the swapping deployment have nearly completed, which refers to the mature stage (II).

Therefore, in two different stages (I and II), the profit functions of the NEV-SM maker under the base model (without the extended warranty service) are formulated, respectively

$$
\begin{gathered}
\pi_{1}^{\mathrm{I}}\left(p_{1}^{\mathrm{I}}, m^{\mathrm{I}}\right)=D_{1}^{\mathrm{I}}\left(p_{1}^{\mathrm{I}}-c\right)-\frac{1}{2} \alpha\left(m^{\mathrm{I}}\right)^{2}+a m^{\mathrm{I}}(17) \\
\pi_{1}^{\Pi}\left(p_{1}^{\Pi}, m^{\Pi}\right)=D_{1}^{\Pi}\left(p_{1}^{\Pi}-c\right)-\frac{1}{2} \alpha\left(m^{\Pi}\right)^{2}+a\left(m^{\Pi}\right)+b\left(m^{\Pi}-m^{\mathrm{I}}\right)
\end{gathered}
$$

and the profit functions of the NEV-SM maker under the extended warranty service model in two different stages (I and II) are shown,

$$
\begin{gathered}
\widetilde{\pi}_{1}^{\mathrm{I}}\left({\widetilde{p_{1}}}^{\mathrm{I}}, \widetilde{m}^{\mathrm{I}}\right)={\widetilde{D_{1}}}^{\mathrm{I}}\left({\widetilde{p_{1}}}^{\mathrm{I}}-c\right)-\frac{1}{2} \alpha\left(\widetilde{m}^{\mathrm{I}}\right)^{2}+a \widetilde{m}^{\mathrm{I}}(19) \\
\widetilde{\pi}^{\Pi}\left({\widetilde{p_{1}}}^{\Pi}, \widetilde{m}^{\Pi}\right)={\widetilde{D_{1}}}^{\Pi}\left({\widetilde{p_{1}}}^{\Pi}-c\right)-\frac{1}{2} \alpha\left(\widetilde{m}^{\Pi}\right)^{2}+a \widetilde{m}^{\Pi}+b\left(\widetilde{m}^{\Pi}-\widetilde{m}^{\mathrm{I}}\right)
\end{gathered}
$$

For the NEV-CM maker, its profit functions remain unchanged. Using the similar method as before, the optimal solutions without/with the extended warranty service are obtained, and the results are shown in Table 2. 
Table 2. The optimal solutions in the presence of subsidies

Without the extended warranty service $\quad$ With the extended warranty service Stage I Stage II

Extended

Stage I Stage II

$\frac{-k\left(2 \alpha \lambda-\delta^{2}-a \delta\right)}{\alpha k^{2}+\beta \delta^{2}-3 \alpha \beta}$

$-k\left(2 \alpha \lambda-\delta^{2}-b \delta\right)$

$\alpha k^{2}+\beta \delta^{2}-3 \alpha \beta \lambda$

$\alpha k^{2}+\beta \delta^{2}-3 \alpha \beta \lambda$

warranty service
duration $T^{* 1, \Pi}$

$-$

$\widetilde{T}^{* \mathrm{I}, \Pi}$

\begin{tabular}{|c|c|c|c|c|c|c|}
\hline $\begin{array}{l}\text { Number } \\
\text { swapping } \\
m^{* 1, \Pi}\end{array}$ & $\begin{array}{r}\text { of } \\
\text { station }\end{array}$ & $\frac{\lambda(3 a+\delta)}{3 \alpha \lambda-\delta^{2}}$ & $\frac{\lambda(3 b+\delta)}{3 \alpha \lambda-\delta^{2}}$ & $\widetilde{m}^{* 1, \Pi}$ & $\frac{k^{2}(a+\delta)-\beta \lambda(3 a+\delta)}{\alpha k^{2}+\beta \delta^{2}-3 \alpha \beta \lambda}$ & $\frac{k^{2}(b+\delta)-\beta \lambda(3 b+\delta)}{\alpha k^{2}+\beta \delta^{2}-3 \alpha \beta \lambda}$ \\
\hline \multirow[b]{2}{*}{ Price } & $p_{1}^{* 1, \Pi}$ & $\frac{\lambda(a \delta+\alpha \lambda)+c\left(3 \alpha \lambda-\delta^{2}\right)}{3 \alpha \lambda-\delta^{2}}$ & $\frac{\lambda(b \delta+\alpha \lambda)+c\left(3 \alpha \lambda-\delta^{2}\right)}{3 \alpha \lambda-\delta^{2}}$ & ${\widetilde{p_{1}}}^{* 1, \Pi}$ & $\begin{array}{c}{\left[-\alpha \beta \lambda(3 c+\lambda)+\alpha k^{2}(c+\lambda)-\beta \delta(a \lambda-c \delta)\right]} \\
/\left(\alpha k^{2}+\beta \delta^{2}-3 \alpha \beta \lambda\right)\end{array}$ & {$\left[-\alpha \beta \lambda(3 c+\lambda)+\alpha k^{2}(c+\lambda)-\beta \delta(b \lambda-c \delta)\right] /\left(\alpha k^{2}+\beta \delta^{2}-3 \alpha \beta \lambda\right)$} \\
\hline & $p_{2}{ }^{* 1, \Pi}$ & $\frac{\lambda\left(2 \alpha \lambda-\delta^{2}-a \delta\right)+c(3 \alpha \lambda}{3 \alpha \lambda-\delta^{2}}$ & $\frac{\lambda\left(2 \alpha \lambda-\delta^{2}-b \delta\right)+c\left(3 \alpha \lambda-\delta^{2}\right)}{3 \alpha \lambda-\delta^{2}}$ & ${\widetilde{p_{2}}}^{* 1, \Pi}$ & $\begin{array}{c}{\left[-2 \alpha \beta \lambda^{2}+c\left(\alpha k^{2}+\beta \delta^{2}\right)+\lambda \beta\left(-3 c \alpha+a \delta+\delta^{2}\right)\right]} \\
/\left(\alpha k^{2}+\beta \delta^{2}-3 \alpha \beta \lambda\right)\end{array}$ & $\begin{array}{c}{\left[-2 \alpha \beta \lambda^{2}+c\left(\alpha k^{2}+\beta \delta^{2}\right)+\lambda \beta\left(-3 c \alpha+b \delta+\delta^{2}\right)\right]} \\
/\left(\alpha k^{2}+\beta \delta^{2}-3 \alpha \beta \lambda\right)\end{array}$ \\
\hline \multirow{2}{*}{ Demand } & $D_{1}^{* 1, \Pi}$ & $\frac{a \delta+\alpha \lambda}{3 \alpha \lambda-\delta^{2}}$ & $\frac{b \delta+\alpha \lambda}{3 \alpha \lambda-\delta^{2}}$ & ${\widetilde{D_{1}}}^{* 1, \Pi}$ & $\frac{-\left(a \beta \delta+\alpha \beta \lambda-\alpha k^{2}\right)}{\alpha k^{2}+\beta \delta^{2}-3 \alpha \beta \lambda}$ & $\frac{-\left(b \beta \delta+\alpha \beta \lambda-\alpha k^{2}\right)}{\alpha k^{2}+\beta \delta^{2}-3 \alpha \beta \lambda}$ \\
\hline & $D_{2}^{* 1, \Pi}$ & $\frac{2 \alpha \lambda-\delta^{2}-a \delta}{3 \alpha \lambda-\delta^{2}}$ & $\frac{2 \alpha \lambda-\delta^{2}-b \delta}{3 \alpha \lambda-\delta^{2}}$ & ${\widetilde{D_{2}}}^{* 1, \Pi}$ & $\frac{-\beta\left(2 \alpha \lambda-\delta^{2}-a \delta\right)}{\alpha k^{2}+\beta \delta^{2}-3 \alpha \beta \lambda}$ & $\frac{-\beta\left(2 \alpha \lambda-\delta^{2}-b \delta\right)}{\alpha k^{2}+\beta \delta^{2}-3 \alpha \beta \lambda}$ \\
\hline \multirow[t]{2}{*}{ Profit } & $\pi_{1}{ }^{* 1, \Pi}$ & $\begin{array}{l}\lambda\left[2(\alpha \lambda)^{2}\right. \\
+\alpha \lambda\left(9 a^{2}+4 a \delta-\delta^{2}\right) \\
\left.-2 a \delta^{2}(2 a+\delta)\right] \\
/\left[2\left(3 \alpha \lambda-\delta^{2}\right)^{2}\right]\end{array}$ & $\begin{aligned} \lambda\left[2(\alpha \lambda)^{2}+\alpha \lambda\right. & \left(9 a^{2}+6 a \delta-\delta^{2}-2 b \delta\right. \\
& \left.+18 a^{2}-18 a b\right) \\
& +6 a b \delta^{2} \\
& \left.-2 \delta^{2}\left(3 a^{2}+a \delta+2 b^{2}\right)\right] \\
& /\left[2\left(3 \alpha \lambda-\delta^{2}\right)^{2}\right]\end{aligned}$ & $\widetilde{\pi}_{1}^{* 1, \Pi}$ & $\begin{aligned}\left\{\lambda \beta^{2}\left[2(\alpha \lambda)^{2}+\right.\right. & \left.\alpha \lambda\left(9 a^{2}+4 a \delta-\delta^{2}\right)-2 a \delta^{2}(2 a+\delta)\right] \\
& -2 \beta k^{2}\left[2(\alpha \lambda)^{2}+\alpha \lambda(a+\delta)(3 a-\delta)\right. \\
& \left.\left.-a \delta^{2}(a+\delta)+\alpha k^{4}\left(a^{2}+2 \alpha \lambda-\delta^{2}\right)\right]\right\} \\
& /\left[2\left(\alpha k^{2}+\beta \delta^{2}-3 \alpha \beta \lambda\right)^{2}\right]\end{aligned}$ & $\begin{aligned}\left\{\alpha \lambda^{2} \beta^{2}\left[-\delta^{2}+\right.\right. & 2 \delta(3 a-b)]+\alpha \lambda^{2} \beta^{2} 9 b^{2}+6 \alpha \beta \lambda k^{2} b^{2} \\
& +2 \alpha \beta \lambda k^{2}\left[\delta^{2}-2 \delta(2 a-b)\right]-\alpha k^{4}\left[\delta^{2}-2 \delta(a-b)\right] \\
& -2 \beta \delta^{2}\left[\beta \lambda\left(a \delta+2 b^{2}\right)\right]+k^{2}\left(a \delta+b^{2}\right) \\
& \left.+2 \lambda \alpha^{2}\left(k^{2}-\beta \lambda\right)^{2}+\alpha k^{4} b^{2}\right\} /\left[2\left(\alpha k^{2}+\beta \delta^{2}-3 \alpha \beta \lambda\right)^{2}\right]\end{aligned}$ \\
\hline & $\pi_{2}{ }^{* 1, \Pi}$ & $\frac{\lambda\left(2 \alpha \lambda-\delta^{2}-a \delta\right)^{2}}{\left(3 \alpha \lambda-\delta^{2}\right)^{2}}$ & $\frac{\lambda\left(2 \alpha \lambda-\delta^{2}-b \delta\right)^{2}}{\left(3 \alpha \lambda-\delta^{2}\right)^{2}}$ & $\widetilde{\pi}_{2}^{* 1, \Pi}$ & {$\left[\beta\left(2 \beta \lambda-k^{2}\right)\left(2 \alpha \lambda-\delta^{2}-a \delta\right)^{2}\right] /\left[2\left(\alpha k^{2}+\beta \delta^{2}-3 \alpha \beta \lambda\right)^{2}\right]$} & {$\left[\beta\left(2 \beta \lambda-k^{2}\right)\left(2 \alpha \lambda-\delta^{2}-b \delta\right)^{2}\right] /\left[2\left(\alpha k^{2}+\beta \delta^{2}-3 \alpha \beta \lambda\right)^{2}\right]$} \\
\hline
\end{tabular}


Lemma 2. Without the extended warranty service,

When $a<\left(\lambda \alpha-\delta^{2}\right) / 2 \delta$ holds on, the optimal price and demand of the NEV-SM maker at stage II are lower than at stageI (i.e., $\left.p_{1}^{\Pi}<p_{1}^{\mathrm{I}}, D_{1}^{\Pi}<D_{1}^{\mathrm{I}}\right)$. When $b<\left(\lambda \alpha-\delta^{2}\right) / 2 \delta$ holds on, we find the optimal price and demand of the NEV-SM maker's rival at stage II are larger than at stageI (i.e., $p_{2}^{\Pi}>p_{2}^{\mathrm{I}}, D_{2}^{\Pi}>D_{2}^{\mathrm{I}}$ ).

The lemma 2 exhibits that in the absence of extended warranty service, when the subsidy standard $a$ and $b$ are below a specific threshold value (i.e., $\left(\lambda \alpha-\delta^{2}\right) / 2 \delta$ ), both the demand and price for the NEV-SM maker at stageI are larger than those at stage II, whereas for the NEV-CM maker, the outcome is opposite, which shows that the government subsidies have little effect on NEV-SM maker at stage II if the government's subsidy size reduce to some degrees. With this in mind, government should still offer sufficient subsidy size to support the NEV-SM makers at stage II, avoiding the investment inefficiency.

Lemma 3. With the extended warranty service,

(i) When $\alpha k^{2}>\beta \delta^{2}+2 a \beta \delta, \lambda>\left(\alpha k^{2}-a \beta \delta\right) / \alpha \beta$ or $\alpha k^{2}<\beta \delta^{2}, \lambda>\left(\beta \delta^{2}-\alpha k^{2}+a \beta \delta\right) / \alpha \beta$ satisfy, for the NEV-SM maker, the optimal price and demand at the preliminary stage I is higher than at the mature stage II (i.e., ${\widetilde{p_{1}}}^{\Pi}<\widetilde{p_{1}}, \widetilde{D_{1}}{ }^{\Pi}<\widetilde{D_{1}}$ );

(ii) when $\lambda \alpha+b \delta>\alpha k^{2} / \beta>\delta^{2}+2 b \delta$ or $\delta^{2}>\alpha k^{2} / \beta>\delta^{2}-\alpha \lambda+b \delta$ hold on, for the NEV-SM maker, the optimal price and demand at the mature stage II is larger than at the preliminary stage I (i.e., ${\widetilde{p_{2}}}^{\Pi}>$ ${\widetilde{p_{2}}}^{\mathrm{I}},{\widetilde{D_{2}}}^{\Pi}>{\widetilde{D_{2}}}^{\mathrm{I}}$.

Lemma 3 reveals that even if the extended warranty services are introduced by the NEV-CM maker, at stage II, government subsidies can help the NEV-CM get better off compared to the stage I. But for the NEV-SM maker, at the initial stage of subsidy, the optimal price keeps lower level, and meanwhile the market shares are larger than its rival, which verify that the government subsidies to the NEV-SM maker play a significant role at stageI when the NEV-CM maker provides the extended warranty services.

Corollary 3. At the stage I (or II),

(i)When $\lambda>\left(\delta^{2}+2 a \delta\right) / \alpha$ (for at the stage $\mathrm{II}, \lambda>\left(\delta^{2}+2 b \delta\right) / \alpha$ ) satisfies, the NEV-SM maker's optimal price and demand in the presence of subsidy at stage I (II) are higher than those in the absence of subsidy, i.e., $p_{1}^{\mathrm{I}}>p_{1}, D_{1}^{\mathrm{I}}>D_{1}\left(p_{1}^{\Pi}>p_{1}, D_{1}^{\Pi}>D_{1}\right)$; while for the NEV-CM maker, the optimal price and demand in the presence of subsidy at stage I (II)are lower than those in the absence of subsidy, i.e., $p_{2}^{\mathrm{I}}<p_{2}, D_{2}^{\mathrm{I}}<D_{2} \quad\left(p_{2}^{\Pi}<p_{2}, D_{2}^{\Pi}<D_{2}\right)$.

(ii) When $\lambda>k^{2} / \beta>\left(\delta^{2}+2 a \delta\right) / \alpha$ or $\frac{\beta \delta^{2}}{2}>\alpha k^{2}>\beta \delta^{2}-\lambda \alpha \beta+2 a \beta \delta$ (for at the stage II, $\alpha k^{2}>\beta \delta^{2}+$ $2 b \beta \delta, \lambda>k^{2} / 2 \beta$ or $\left.\alpha k^{2}<\beta \delta^{2}, \lambda>\left(\beta \delta^{2}-\alpha k^{2}+2 b \beta \delta\right) / \alpha \beta\right)$ holds on, the NEV-SM maker's optimal price and demand in the presence of subsidy at stage I (II) are lower than those in the absence of subsidy, i.e., $\widetilde{p_{1}}{ }^{\Pi}<$ $\widetilde{p_{1}}, \widetilde{D_{1}}<\widetilde{D_{1}}\left(\widetilde{p_{1}}{ }^{\Pi}<\widetilde{p_{1}}, \widetilde{D_{1}}{ }^{\Pi}<\widetilde{D_{1}}\right)$; while for the NEV-CM maker, the optimal price, the duration of extended warranty service and demand in the presence of subsidy at stage I (II)are higher than those in the absence of subsidy, i.e., ${\widetilde{p_{2}}}^{\mathrm{I}}>\widetilde{p_{2}},{\widetilde{D_{2}}}^{\mathrm{I}}>\widetilde{D_{2}}, \widetilde{T}^{\mathrm{I}}>\widetilde{T}\left(\widetilde{p_{2}}{ }^{\Pi}>\widetilde{p_{2}},{\widetilde{p_{2}}}^{\Pi}>\widetilde{D_{2}}, \widetilde{T}^{\Pi}>\widetilde{T}\right)$.

Corollary 3 (i) illustrates that when $\lambda$ surpasses a specific value, government subsidy can help the NEV-SM maker promote swapping mode, but thwart charging mode to widely be adopted at both stage. Interestingly, even if the NEV-SM maker's optimal price in the presence of subsidy are higher than that in the absence of subsidy, but the demand still expands in the presence of subsidy. Corollary 3

(ii) shows an opposite side, that is when $\lambda>k^{2} / \beta>\left(\delta^{2}+2 a \delta\right) / \alpha$ or $\frac{\beta \delta^{2}}{2}>\alpha k^{2}>\beta \delta^{2}-\lambda \alpha \beta+2 a \beta \delta$,

government subsidy policy is beneficial to the NEV-CM maker rather than the NEV-SM maker, which reveals that such policy is a two-edged sword, and remind policymaker to tradeoff between swapping mode and charging mode in the development of NEV. 


\section{Numerical study}

In this section, we further investigate the effect of battery swapping and charging modes on consumer surplus, and social welfares as well in the setting of subsidies, then examine the impact of key variables, including swapping station's convenience coefficient, and the coefficient of duration of extended warranty service, on the production decision of the two NEV maker, and consumers' choice between battery swapping and charging modes under different conditions (without/with the extended warranty service, with/without subsides).

Based on the literature (Chediak, 2017; Widrick, 2018) and actual data from Nio Auto and BAIC, we could set $\lambda$ as 0.7 , the unit cost of the NEV-SM and NEV-CM is 1 , the actual value of the products is 0.5 , the service coefficient of the extended warranty service is 0.2 to 0.6 , and then we assume the government subsidy under stage I and II $a=0.4$ and $b=0.1$. As Nio Auto officially announced the power swapping technology can realize the rapid replacement of power batteries within 3 minutes. Hence, the battery swapping station convenience coefficient ranges from 0.5 to 0.9 . The other specific parameter values are shown in the following Table 3.

Table 3. The value of parameters

\begin{tabular}{ccccccccc}
\hline$c$ & $v$ & $k$ & $a$ & $b$ & $\delta$ & $\alpha$ & $\beta$ & $\lambda$ \\
1 & 0.5 & $0.2-0.6$ & 0.4 & 0.1 & $0.5-0.9$ & $0.6-0.8$ & $0.3-0.5$ & 0.7 \\
\hline
\end{tabular}

\subsection{The impact on consumer surplus and social welfare}

In this sub-section we examine the effect of two key exogenous variables (i.e., $\delta, k$ ) on the consumer surplus and social welfare under different conditions (without/with the extended warranty service, with/without subsides). For a consumer with NEV-SM or NEV-CM, the surplus for the stage I is as follows:

$$
\operatorname{CS}^{\mathrm{I}}\left(p_{1}{ }^{\mathrm{I}}, m^{\mathrm{I}}\right)=\left(v-p_{1}{ }^{\mathrm{I}}+\delta m^{\mathrm{I}}\right) \times \frac{\delta m^{\mathrm{I}}-p_{1}{ }^{\mathrm{I}}+p_{2}{ }^{\mathrm{I}}}{\lambda}+\left[v(1+\lambda)-p_{2}{ }^{\mathrm{I}}\right] \times\left(1-\frac{\delta m^{\mathrm{I}}-p_{1}{ }^{\mathrm{I}}+p_{2}{ }^{\mathrm{I}}}{\lambda}\right)
$$

For social welfare, it consists of three facets: the profit from the NEV-SM maker and the NEV-CM maker, the government subsidy and the consumers' surplus, thus social welfare for the stage I can be described as follows:

$$
\begin{aligned}
& S W^{\mathrm{I}}\left(p_{1}{ }^{\mathrm{I}}, m^{\mathrm{I}}\right)=\underbrace{\frac{\delta m^{\mathrm{I}}-p_{1}{ }^{\mathrm{I}}+p_{2}{ }^{\mathrm{I}}}{\lambda} \times\left(p_{1}{ }^{\mathrm{I}}-c\right)-\frac{1}{2} \alpha\left(m^{\mathrm{I}}\right)^{2}+a m^{\mathrm{I}}}_{\text {The profit of the NEV-SM }}+\underbrace{\left(1-\frac{\delta m^{\mathrm{I}}-p_{1}{ }^{\mathrm{I}}+p_{2}{ }^{\mathrm{I}}}{\lambda}\right) \times\left(p_{2}{ }^{\mathrm{I}}-c\right)}_{\text {The profit of the NEV-CM }}- \\
& \underbrace{a m_{s} \mathrm{I}}_{\text {The government subsidy on stage I }}+\underbrace{C S^{\mathrm{I}}}_{\text {The consumers' surplus on stage I }}
\end{aligned}
$$

Similar to the formation (21) and (22), we can obtain the corresponding consumer surplus and social welfare under other different conditions. For the brevity, we skip it. 


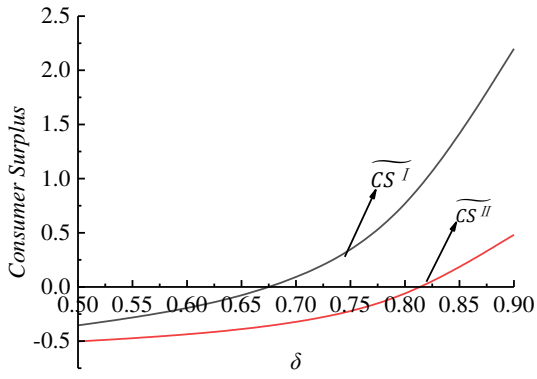

(a)

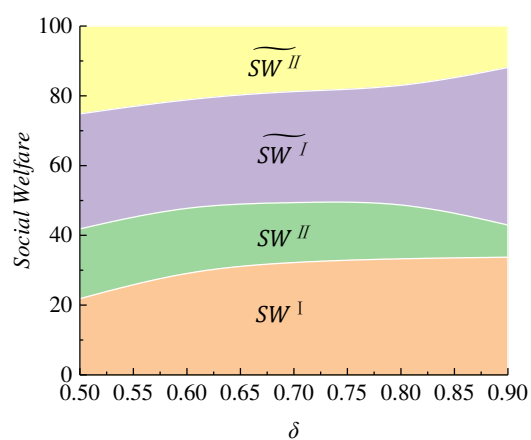

(c)

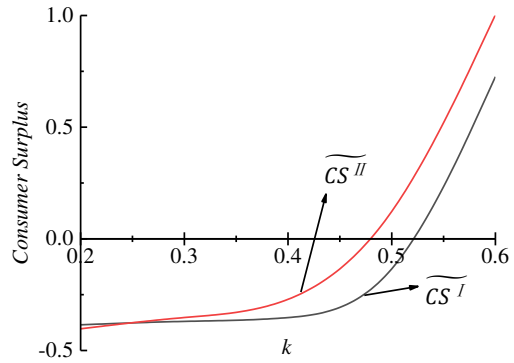

(b)

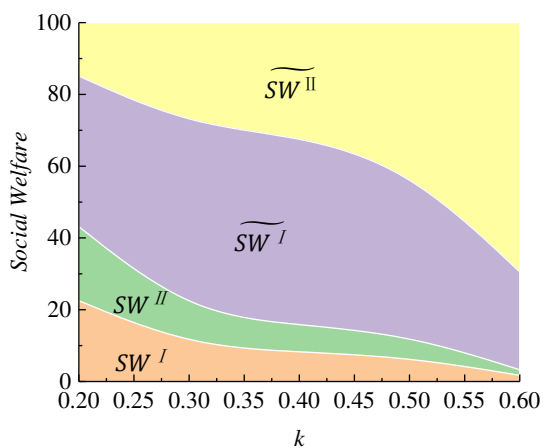

(d)

Fig.1 The influence on consumer surplus and social welfare

Fig. 1(a) compares the effect of convenience coefficient of swapping stations on consumer surplus at stage I and II under the extended warranty service. It reveals that there exist a threshold value $(\delta=$ 0.84), above which the consumer surplus at stage II is larger than zero, hence consumer intend to purchase, otherwise, consumer will give up. Meanwhile, it is obvious that the consumer surplus at stage I is more than at stage II. This means that the higher the convenience of a power swapping station, the higher the consumer surplus, and the less effective government subsidies. Fig. 1(b) shows the effect of extended warranty service coefficient on consumer surplus. With the increase of $k$, the consumer surplus at stage I and II increase, this means that the higher the service level, the higher the consumer surplus at both stages, but the consumer surplus at stage II is more than at stage I, which is opposite to Fig. 1(a).

Fig. 1(c)(d) demonstrates that the difference in social welfare at stage I and II with/without the extended warranty service under the influence of $\delta$ and $k$. We find that regardless of the change of $\delta$ or $k$, the social welfare at stage II with the extended warranty service is largest, followed by that at stage I with the extended warranty service, the smallest social welfare is the case neither subsidy nor the extended warranty service.

\subsection{The impact on performance}

The numerical examples examine the single and joint effects of critical parameters on profits, respectively, in different conditions (without/with the extended warranty service, at stage I/II). To exclude the influence of external factors, other variables are taken to have a moderate value.

(1) The single effect of $\alpha$ and $\delta$ on profits 


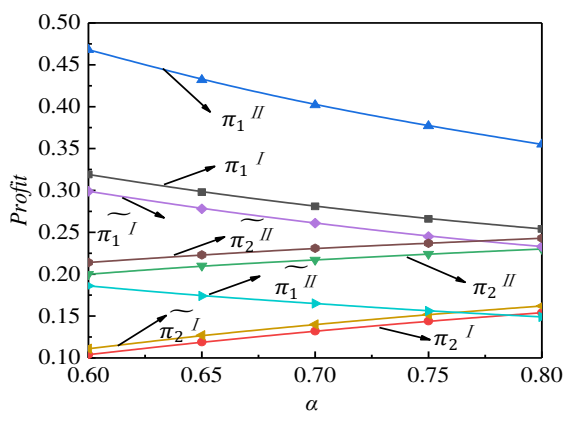

(a)

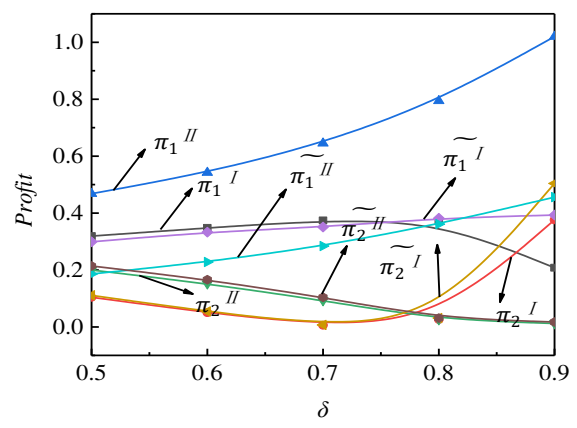

(b)

Fig. 2 The single effect of $\alpha$ and $\delta$

Fig. 2(a) exhibits that no matter what condition is, when the cost coefficient of swapping station increases, the NEV-SM maker's profit decreases, while the NEV-CM maker's profit increases. Meanwhile, comparing the profits of the two makers, we find the profit of the NEV-SM maker is the largest when the NEV-CM maker does not provide the extended warranty service, and swapping network has taken shapes, whereas the profit of NEV-CM maker is the lowest when the NEV-CM maker does not provide the extended warranty service, and swapping network is at initial stage.

Fig. 2(b) shows that no matter what condition is, the NEV-SM maker's profit increases with $\delta$, while the NEV-CM maker's profit drops in $\delta$. This means that the convenience of power swapping has brought considerable profit to the NEV-SM maker, and yet has an opposite effect on its competitor. From Fig. 2(b) we also observe that the NEV-SM maker is most profitable when the NEV-CM maker does not provide the extended warranty service, and swapping network is at the maturity stage.

(2) The single effect of $\beta$ and $k$ on profits

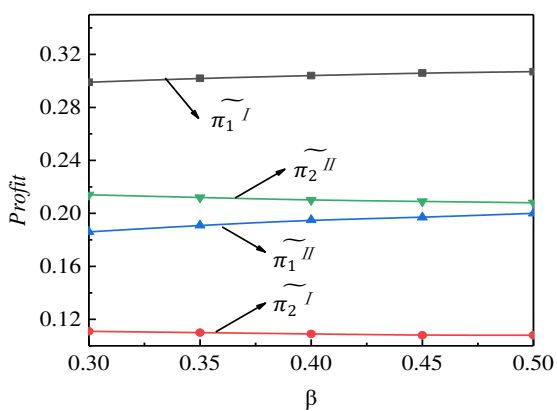

(a)

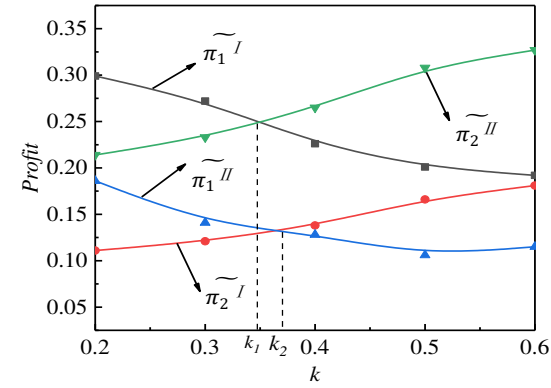

(b)

Fig. 3 The single effect of $\beta$ and $k$

Fig. 3(a) shows that in the setting of warranty service and subsidy, the NEV-SM maker's profit increases slightly and yet the NEV-CM one's profit decreases a little with $\beta$. Although the increase of cost efficient of the extended warranty service bring about the related change in two makers' profits, such changes do not show obvious, which means the cost efficient of the extended warranty service is not sensitive factor for NEV makers.

Fig. 3(b) displays that in the setting of warranty service and subsidy, the profit of the NEV-SM maker decreases in $k$, whereas the profit of the NEV-CM maker increases with $k$. It implies that the increase of extended warranty service coefficient can enhance the NEV-CM maker's profitability, which in turn impairs the NEV-SM' maker's performance. From Fig. 3(b), there exist a specific value $k_{1}$, below 
which the lowest profit occurs to the NEV-CM maker in the case where the swapping network has taken shape, otherwise, it happens when the swapping network is at an infant stage. In contrast, there is another certain value $k_{2}$, above which the highest profit occurs to the NEV-SM maker in the case where the swapping network has taken shape, otherwise, it happens when the swapping network is at an initial stage.

\section{(3) The joint effects on profits}

In the previous analysis, we further explore the joint effects of $\delta$ and $k$ on profits of the two makers, with extended warranty service in two stages, namely, under the preliminary stage (stage I) and the mature stag (stage II), and sets up two cases about the low $(\alpha / \beta=3)$ and high $(\alpha / \beta=1)$ marginal cost ratio.

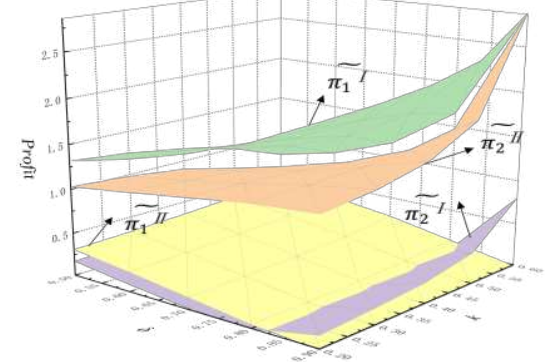

(a) $\alpha / \beta=3$

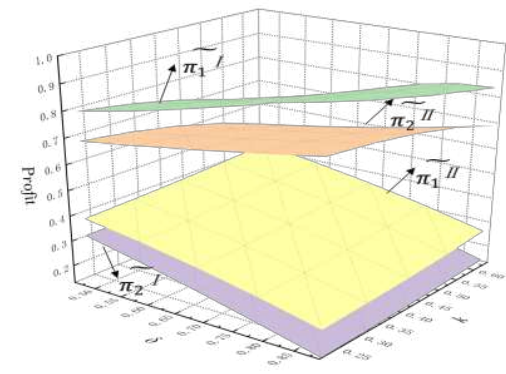

(b)

$$
\alpha / \beta=1
$$

Fig.4 The joint effects of $\delta$ and $k$

Fig. 4(a) shows the NEV-SM maker's profit increases at stage $\mathrm{I}$ and yet decreases with $\delta$ and $k$ at stage II, while the NEV-CM maker's profit first decreases and then arises with $\delta$ and $k$ at stage I, but its continue to increase with $\delta$ and $k$ at stage II. Overall, the NEV-SM maker's profit at stage I is always higher than the NEV-CM maker's profit at stage I or II. Fig. 4(b) demonstrates the similar pattern, the only difference is that when the marginal cost ratio. is higher $(\alpha / \beta=3)$, its impact on the NEV-SM maker and the NEV-CM maker is relatively larger, while the marginal cost ratio is relatively smaller $\quad(\alpha / \beta=1)$, the profit of both carmakers does not change much.

\section{Conclusions}

Due to the limited capacity of batteries, battery swapping mode (SM) and charging mode (CM) can be offered by NEV makers to recharge, thus will inevitably influence potential consumers' purchase behaviors accordingly. To examine the impact of SM and CM on consumers' purchase intention, this paper examines a duopolistic market consisting of two new energy vehicle makers (i.e., NEV-SM maker and NEV-CM maker), who adopting SM and CM to service consumers, respectively. Considering SM characterized with low initial investment and ease of use for consumers, NEV-CM makers capitalize on battery extended warranty service in response to rivals' utilization of SM, thereby non-cooperative game models are formulated, in which government subsidies are taken into account, both the optimal decision for the NEV-SM maker and NEV-CM maker are analyzed under three scenarios: without extended warranty service, with extended warranty service, and with extended warranty service and subsidy. Our results offer some insights as follows.

(1) In the absence of extended warranty service, there exists an optimal equilibrium condition for both carmakers, in which the NEV-SM maker adopts the low-price strategy at the initial development 
stage of NEV-SM, with aims to tap into the market and attract potential consumers, yet this move is not a long-term solution due to that the NEV-CM maker invariably occupies more market shares and profits in the NEV market.

(2) In the presence of extended warranty service, the NEV-CM maker does not necessarily dominates the market. Consumers' choice of two kinds of products relies on two critical factors, namely, $\lambda$ and $\frac{\delta}{k}$. When $0<\frac{\delta}{k}<(2 \alpha / \beta)^{1}, \lambda>k^{2} / \beta$, the NEV-SM maker's profit does surpass the NEV-CM maker's. Nevertheless, the extended warranty service ultimately enhances the NEV-CM maker's dominance.

(3) In the absence of extended warranty service, when the subsidy standard $a$ and $b$ are below a specific threshold value (i.e., $\left(\lambda \alpha-\delta^{2}\right) / 2 \delta$ ), both the demand and price for the NEV-SM maker at stageI are larger than those at stage II, whereas for the NEV-CM maker, the outcome is opposite, which shows that the government subsidies have little effect on NEV-SM maker at stage II if the government's subsidy size reduce to some degrees.

(4) In the presence of extended warranty service, government subsidy can dramatically increase the NEV-SM maker's market shares at stageI. Meanwhile, the numerical studies show that the NEV-SM maker's profit, consumer surplus and social welfare have been improved by implementing subsidy policy, which implies that subsidy is a critical factor in propelling the diffusion of swapping mode at the initial stage.

There is still much space for future research. Our paper only examines NEV makers, and did not extend to a supply chain system composed of makers and suppliers, thus one of the possible directions is to investigate optimal power swapping stations and extended warranty services when taking investment sharing into account. Moreover, replacement and trade-in activity of products is a normal phenomenon in NEV market, and thus it would be interesting to examine optimal decisions in the context of replacement and trade-in strategy.

\section{Appendix}

Table 1 Summary of notations.

\begin{tabular}{cl}
\hline Notations & Definition \\
\hline Decisions & \\
$m$ & The number of battery swapping stations \\
$T$ & The duration of extended warranty service \\
$p_{i}$ & Product price of NEVs with $i$ mode ( $i=1$ refers to SM, $i=2$ stands for CM) \\
Parameters & \\
$\alpha$ & The cost coefficient of battery swapping stations \\
$\beta$ & The cost coefficient of extended warranty service \\
$\delta$ & The battery swapping station's convenience coefficient \\
$k$ & The extended warranty service coefficient \\
$\lambda$ & The valuation incremental rate of CM relative to SM \\
$s$ & The amount of government subsidy \\
$u_{i}$ & Consumer utility of NEVs with $i$ mode $(i=1$ refers to SM, $i=2$ stands for CM) \\
$v$ & Consumer valuation of product of NEV-SM \\
$c_{i}$ & Unit product cost of NEVs with $i$ mode $(i=1$ refers to SM, $i=2$ stands for CM) \\
$D_{i}$ & Consumer demand of NEVs with $i$ mode $(i=1$ refers to $S M, i=2$ stands for CM) \\
$\pi_{i}$ & Profit of NEVs with $i$ mode $(i=1$ refers to $S M, i=2$ stands for CM) \\
\hline
\end{tabular}


Proof of Proposition 1. The first-order derivatives of Equation (9) with respect to $p_{1}$ and $m$ can be set to 0 as follows:

The Hessian matrix of Equation (9) is:

$$
\begin{aligned}
& \frac{\partial \pi_{1}}{\partial p_{1}}=\frac{c-2 p_{1}+p_{2}+\delta m}{\lambda}=0 \\
& \frac{\partial \pi_{1}}{\partial m}=\frac{\delta\left(p_{1}-c\right)-\lambda \alpha m}{\lambda}=0
\end{aligned}
$$

$$
H\left(p_{1}, m\right)=\left[\begin{array}{cc}
\frac{\partial^{2} \pi_{1}}{\partial p_{1}^{2}} & \frac{\partial^{2} \pi_{1}}{\partial p_{1} \partial m} \\
\frac{\partial^{2} \pi_{1}}{\partial m \partial p_{1}} & \frac{\partial^{2} \pi_{1}}{\partial m^{2}}
\end{array}\right]=\left[\begin{array}{cc}
\frac{-2}{\lambda} & \frac{\delta}{\lambda} \\
\frac{\delta}{\lambda} & -\alpha
\end{array}\right]
$$

Note that the Hessian matrix is a negative definite for all decision variables that satisfy the conditions: $\lambda>\frac{\delta^{2}}{2 \alpha}$. The first-order derivatives of Equation (9) with respect to $p_{2}$ can be set to 0 as follows:

$$
\frac{\partial \pi_{2}}{\partial p_{2}}=\frac{\lambda+c-2 p_{2}+p_{1}-\delta m}{\lambda}=0
$$

Note that $\frac{\partial^{2} \pi_{2}}{\partial p_{2}{ }^{2}}=\frac{-2}{\lambda}<0$, since the second-order partial derivative is a joint concave function, there is always a maximum. Solving Equations (9)-(10) and (A1)-(A2), we derive the results of Proposition 1.

Proof of Lemma 1. Considering the negative conditions of the Hessian matrix in Equations (A3), we obtain that $p_{1}, p_{2}$ and $m$ are positive when $\lambda>\frac{\delta^{2}}{2 \alpha}$. For further guaranteeing the demand NEV-SM and NEV-CM positive, the inequality $\frac{2 \alpha \lambda-\delta^{2}}{3 \alpha \lambda-\delta^{2}}>0$ holds on. It is easy to explain that when $p_{1}<p_{2}, \lambda>\frac{\delta^{2}}{2 \alpha^{\prime}}$, the conditions of optimal solutions under the base model is given by $\lambda>\frac{\delta^{2}}{\alpha}$, whereas if $\frac{\delta^{2}}{\alpha}>\lambda>\frac{\delta^{2}}{2 \alpha}$ satisfies, the optimal solutions do not exist.

Proof of Theorem1. Similar to Lemma 1, and we obtain the conditions under base model by $\lambda>\frac{\delta^{2}}{\alpha}$. Recall the optimal values $p_{1}{ }^{*}, p_{2}{ }^{*}, D_{1}{ }^{*}, D_{2}{ }^{*}$ and $m^{*}$ in Table 2 and compare the demand and profit between NEV-SM and NEV-CM that is, $D_{1}{ }^{*}-D_{2}{ }^{*}=\frac{-\alpha \lambda+\delta^{2}}{3 \alpha \lambda-\delta^{2}}<0$, this inequality is always true as long as the limiting conditions are satisfied and the demand of NEV-CM exceeds to NEV-SM. Furthermore, the profit of NEV-SM and NEV-CM can be recognized by $\pi_{1}{ }^{*}=\frac{\alpha \lambda^{2}\left(2 \alpha \lambda-\delta^{2}\right)}{2\left(3 \alpha \lambda-\delta^{2}\right)}$ and $\pi_{2}{ }^{*}=\frac{\lambda\left(2 \alpha \lambda-\delta^{2}\right)^{2}}{3 \alpha \lambda-\delta^{2}}$. The profit point for NEV-SM and NEV-CM under the base model can be derived by: $\pi_{1}{ }^{*}-\pi_{2}{ }^{*}=$ $\frac{\lambda\left(-6 \alpha^{2} \lambda^{2}+7 \alpha \lambda \delta^{2}-2 \delta^{4}\right)}{2\left(3 \alpha \lambda-\delta^{2}\right)^{2}}<0$. Conclusively, we obtain that when $\lambda>\frac{\delta^{2}}{\alpha}$, the NEV-SM gain less profits than NEV-CM under the base model.

Proof of Corollary 1. Recall the optimal profit of NEV-SM in Proposition 1, that is, $\pi_{1}{ }^{*}=\frac{\alpha \lambda^{2}\left(2 \alpha \lambda-\delta^{2}\right)}{2\left(3 \alpha \lambda-\delta^{2}\right)}$, we can easily find the relationship: when the condition $\lambda>\frac{\delta^{2}}{2 \alpha}$ is satisfied, $\frac{\partial \pi_{1}{ }^{*}}{\partial \alpha}=\frac{-\lambda^{2} \delta^{2}\left(\alpha \lambda-\delta^{2}\right)}{2\left(3 \alpha \lambda-\delta^{2}\right)^{3}}<0$,and $\frac{\partial \pi_{1}{ }^{*}}{\partial \delta}=\frac{\alpha \delta \lambda^{2}\left(\alpha \lambda-\delta^{2}\right)}{\left(3 \alpha \lambda-\delta^{2}\right)^{3}}>0$. According to these inequation, it is sufficient for us to illustrate the findings in Corollary 1.

Proof of Proposition 2. Similar to Proposition 1, taking the first order derivatives of Equation (14) with respect to $\widetilde{p_{1}}$ and $\widetilde{m}$ making them equal to 0 , we obtain:

$$
\begin{aligned}
& \frac{\partial \widetilde{\pi_{1}}}{\partial \widetilde{p_{1}}}=\frac{c-k \tilde{T}-2 \widetilde{p_{1}}+\widetilde{p_{2}}+\delta \widetilde{m}}{\lambda}=0 \\
& \frac{\partial \widetilde{\pi_{1}}}{\partial \widetilde{m}}=\frac{\delta\left(\widetilde{p_{1}}-c\right)-\lambda \alpha \widetilde{m}+\alpha \lambda}{\lambda}=0
\end{aligned}
$$

The Hessian matrix of Equation (14) is: 


$$
H\left(\widetilde{p_{1}}, \widetilde{m}\right)=\left[\begin{array}{cc}
\frac{\partial^{2} \widetilde{\pi_{1}}}{\partial \widetilde{p}_{1}^{2}} & \frac{\partial^{2} \widetilde{\pi_{1}}}{\partial \widetilde{p_{1}} \partial \widetilde{m}} \\
\frac{\partial^{2} \widetilde{\pi_{1}}}{\partial \widetilde{m} \partial \widetilde{p_{1}}} & \frac{\partial^{2} \widetilde{\pi_{1}}}{\partial \widetilde{m}^{2}}
\end{array}\right]=\left[\begin{array}{cc}
\frac{-2}{\lambda} & \frac{\delta}{\lambda} \\
\frac{\delta}{\lambda} & -\alpha
\end{array}\right]
$$

Note that the Hessian matrix of $\widetilde{\pi_{1}}$ is a negative definite only for all decision variables that satisfy the conditions: $\lambda>\frac{\delta^{2}}{2 \alpha}$. Similar to Equation (14), we omit the specific calculation process of Equation (15), the conditionssatisfied by the Hessiannegative definite is: $\lambda>\frac{k^{2}}{2 \beta}$. To sum up, the conditions are that, when $\alpha k^{2}>\beta \delta^{2}, \lambda>k^{2} / 2 \beta$; while $\alpha k^{2}<\beta \delta^{2}, \lambda>\delta^{2} / 2 \alpha$.By Solving Equations (14)-(15) and (A7), we accordingly deduce the optimal solution of Proposition 2 .

Proof of Theorem 2. We first verify our findings in Theorem 2(i), and considering the negative conditions satisfied by the Hessian matrix in Equations (A7) and for further guaranteeing the demands are positive, we let ${\widetilde{D_{1}}}^{*}=\frac{\alpha k^{2}-a \beta \delta-\alpha \beta \lambda}{\alpha k^{2}-3 \alpha \beta \lambda+\beta \delta^{2}}>0$ and ${\widetilde{D_{2}}}^{*}=\frac{\beta \delta^{2}+a \beta \delta-2 \alpha \beta \lambda}{\alpha k^{2}-3 \alpha \beta \lambda+\beta \delta^{2}}>0$. By solving $\lambda$ in these equations, we have the results that is, when $2 \alpha k^{2}>\beta \delta^{2}, \lambda>k^{2} / \beta$; while $2 \alpha k^{2}<\beta \delta^{2}, \lambda>\delta^{2} / 2 \alpha$. We compare the optimal demand and profit of NEV-SM and NEV-CM with the limiting conditions under the extended warranty service, and know that, ${\widetilde{D_{1}}}^{*}-{\widetilde{D_{2}}}^{*}=\frac{\alpha k^{2}-2 a \beta \delta+\alpha \beta \lambda-\beta \delta^{2}}{\alpha k^{2}-3 \alpha \beta \lambda+\beta \delta^{2}} \quad, \quad \widetilde{\pi}_{1}^{*}-\widetilde{\pi}^{*}=$ $\frac{\left(2 \alpha \lambda-\delta^{2}\right)\left(\alpha k^{4}-3 \alpha \beta^{2} \lambda^{2}+2 \beta^{2} \delta^{2} \lambda-\beta k^{2} \delta^{2}\right)}{2\left(\alpha k^{2}-3 \alpha \beta \lambda+\beta \delta^{2}\right)^{2}}$. Hence, the relationships of demand and profit of NEV-SM and NEV-CM are determined by: when $2 \alpha k^{2}>\beta \delta^{2}, \lambda>k^{2} / \beta$, we obtain ${\widetilde{D_{1}}}^{*}<{\widetilde{D_{2}}}^{*},{\widetilde{\pi_{1}}}^{*}>{\widetilde{\pi_{2}}}^{*}$, the findings in Theorem 2(i) can be proved. When $2 \alpha k^{2}<\beta \delta^{2}, \delta^{2} / 2 \alpha<\lambda<\left(\beta \delta^{2}-\alpha k^{2}\right) / 2 \alpha \beta$, the relationships do not exist, then when $2 \alpha k^{2}<\beta \delta^{2}, \lambda>\left(\beta \delta^{2}-\alpha k^{2}\right) / 2 \alpha \beta$, we deduce that ${\widetilde{D_{1}}}^{*}<{\widetilde{D_{2}}}^{*}, \widetilde{\pi}_{1}^{*}>\widetilde{\pi}_{2}^{*}$. That means the demand of $\mathrm{SM}$ is always lower than $\mathrm{CM}$, while the relationships of profit between them depends on the limitations. For the reason that, Theorem 2(ii) can be elaborated.

Proof of Corollary2. Resemble to Corollary 1, we recall the optimal extended warranty service and the number of swapping station in the setting of extended warranty service, that is, $\tilde{T}^{*}=$ $\frac{k\left(a \delta-2 \alpha \lambda+\delta^{2}\right)}{\alpha k^{2}-3 \alpha \beta \lambda+\beta \delta^{2}}, \widetilde{m}^{*}=\frac{\beta \lambda(3 a+\delta)-k^{2}(a+\delta)}{\alpha k^{2}-3 \alpha \beta \lambda+\beta \delta^{2}}$. We can easily get the monotonicity of $\delta$, Corollary 2(i) can be validated when $2 \alpha k^{2}>\beta \delta^{2}, \lambda>k^{2} / \beta$ by letting $\frac{\partial \widetilde{T}^{*}}{\partial \delta}=\frac{2 \alpha k \delta\left(k^{2}-\beta \lambda\right)}{\alpha k^{2}-3 \alpha \beta \lambda+\beta \delta^{2}}>0, \frac{\partial \widetilde{m}^{*}}{\partial \delta}=\frac{\left(k^{2}-\beta \lambda\right)\left(\alpha k^{2}-3 \alpha \beta \lambda-\beta \delta^{2}\right)}{\alpha k^{2}-3 \alpha \beta \lambda+\beta \delta^{2}}>0$. While $2 \alpha k^{2}<\beta \delta^{2}, \lambda>\left(\beta \delta^{2}-\alpha k^{2}\right) / 2 \alpha \beta$, we get $\frac{\partial \tilde{T}^{*}}{\partial \delta}<0, \frac{\partial^{*}}{\partial \delta}<0$. Thus, the findings in Corollary 2(ii) can be illustrated.

Proof of Lemma 2. Using the same method as 1, we can obtain the limit condition is $\lambda>\left(\delta^{2}+2 \alpha \delta\right) / \alpha$, and similar to Proposition 1 and 2, we can discover that the optimal solution of NEV-SM and NEV-CM, the specific solutions is shown in Table 2. Through the inequality, $p_{1}^{\mathrm{II}}-p_{1}^{\mathrm{I}}=\frac{\lambda \delta(b-a)}{3 \lambda \alpha-\delta^{2}}<0, p_{2}^{\mathrm{II}}-p_{2}^{\mathrm{I}}=$ $\frac{\lambda \delta(a-b)}{3 \lambda \alpha-\delta^{2}}>0, D_{1}^{\mathrm{II}}-D_{1}^{\mathrm{I}}=\frac{\delta(b-a)}{3 \lambda \alpha-\delta^{2}}<0, D_{2}^{\mathrm{II}}-D_{2}{ }^{\mathrm{I}}=\frac{\delta(a-b)}{3 \lambda \alpha-\delta^{2}}>0$,we can see clearly about the changes in price and demand of NEV-SM and NEV-CM in the absence of extended warranty with different government subsidies.

Proof of Lemma 3. The proof of Lemma 3 is similar to Lemma 2, we will ellipsis the proof here and do not explain specific any more.

Proof of Corollary 3. Firstly, we will verify $p_{1}{ }^{\mathrm{I}}-p_{1}=\frac{a \lambda \delta}{3 \lambda \alpha-\delta^{2}}>0, p_{2}{ }^{\mathrm{I}}-p_{2}=\frac{-a \lambda \delta}{3 \lambda \alpha-\delta^{2}}<0, D_{1}{ }^{\mathrm{I}}-D_{1}=$ $\frac{a \delta}{3 \lambda \alpha-\delta^{2}}>0, D_{2}{ }^{\mathrm{I}}-D_{2}=\frac{-a \delta}{3 \lambda \alpha-\delta^{2}}<0$. From Table 2, we understand the price and demand of NEV-CM under different situations. By detecting the intersection with respect to $\lambda$ of the inequality and the limiting 
conditions $\left[\alpha k^{2}>\left(\delta^{2}+2 a \delta\right) / \alpha\right]$, we know about the Corollary 3(i) is always right. Table 2 shows the retail price of NEV-SM and NEV-CM under the preliminary stage of government subsidy, it is simply to attain the different cases, which can be expressed by $\widetilde{p_{1}^{I}}-\widetilde{p_{1}}=\frac{-a \beta \delta \lambda}{\alpha k^{2}-3 \alpha \beta \lambda+\beta \delta^{2}}<0, \widetilde{p_{2}}-\widetilde{p_{2}}=$ $\frac{a \beta \delta \lambda}{\alpha k^{2}-3 \alpha \beta \lambda+\beta \delta^{2}}>0, \widetilde{D_{1}^{\mathrm{I}}}-\widetilde{D_{1}}=\frac{-a \beta \delta}{\alpha k^{2}-3 \alpha \beta \lambda+\beta \delta^{2}}<0, \widetilde{D_{2}^{\mathrm{I}}}-\widetilde{D_{2}}=\frac{a \beta \delta}{\alpha k^{2}-3 \alpha \beta \lambda+\beta \delta^{2}}>0$. By solving the intersection with respect to $\lambda$ in these inequations $\left[\alpha k^{2}>\beta \delta^{2}+2 a \beta \delta, \lambda>k^{2} / \beta\right.$ or $2 \alpha k^{2}<\beta \delta^{2}, \lambda>\left(\beta \delta^{2}-\alpha k^{2}+\right.$ $2 a \beta \delta) / \alpha \beta$ ], we get results the above relationship is always true so long as the limiting conditions are satisfied. The Corollary 3(ii) can be henceforth demonstrated. For the stage II, the results can be verified by the same method.

\section{Declarations}

- Ethics approval and consent to participate

Not applicable

- Consent for publication

The authors declare that they are consent for publication.

- Availability of supporting data

Not applicable

- Competing interests

The authors declare that they have no competing interests.

- $\quad$ Funding

National Natural Science Foundation of China $(71872076,71964023)$

- Authors' contributions

The article was jointly prepared by all authors. All authors read and approved the final manuscript.

- Acknowledgements

Thank for anyone's helps for improving this manuscript.

\section{References}

Liu, X.. Wu W., Y.. Zou, H. Zhang, L.. Yao,. (2016). Economic dispatch for microgrid with electric vehicles inplug-in charging and battery swapping modes. in Proc. IEEE PES Asia-Pacific Power Energy Eng. Conf., Xi'an, China, Oct. 1158-1163.

Gallego, G., Wang, R., Hu, M., Ward, J., \& Beltran, J. L. (2015). No claim? Your gain: Design of residual value extended warranties under risk aversion and strategic claim behavior. Manufacturing $\mathcal{E}$ Service Operations Management, 17(1), 87-100.

He, Z., Huang, D. L., \& He, S. G. (2018). Design of extended warranty service in a dual supply channel. Total Quality Management, 6(18), 1-18.

Hof, J., Schneider, M., \& Goeke, D. (2017). Solving the battery swap station location-routing problem with capacitated electric vehicles using an AVNS algorithm for vehicle-routing problems with intermediate stops. Transp. Res. B, Methodol., 97(5), 102-112.

Li J., Ku Y., Liu C., Zhou Y. (2019). Dual credit policy: promoting new energy vehicles with battery recycling in a competitive environment. Journal of Cleaner Production, doi.org/10.1016/j.jclepro.2019.118456

Li J., Ku Y., Yu Y., Liu C. (2020). Optimizing production of new energy vehicles with across chain cooperation under China's dual credit policy. Energy, 2:1330-1340.

Liu, W.X., Yi, H. T. (2020). What Affects the Diffusion of New Energy Vehicles Financial Subsidy Policy? 
Evidence from Chinese Cities. International Journal of Environmental Research and Public Health, 17(3), 726-729. doi: 10. 3390/ ijerph17030726.

Kurata, H., \& Nam, S. H. (2010). After-sales service competition a supply chain: Optimization of customer satisfaction level or profit or both? Internet Journal of Production Economics, 127(1), 136-146.

Lau, HS., Su, C., Wang, Y. Y., \& Hua, Z. S. (2012). Volume discounting coordinates a supply chain effectively when demand is sensitive to both price and sales effort. Computer Opera Res, 39(12), 3267-80.

Ma, J. H., Ai, X. Z., \& Tang, X. W. (2015). Vertical channel structure choices for competing supply chains with extended warranty services. Journal of Systems Engineering, 30(4), 539-553.

Chediak, M. (2017). The Latest Bull Case for Electric Cars: The Cheapest Batteries Ever. https://www.bloomberg.com/news/articles/20171205/latest

Moghaddam, Z., Ahmad, I., Habibi, D., \& Phung, Q. V. (2018). Smart Charging Strategy for Electric Vehicle Charging Stations. IEEE Trans. Transport. Electric., 4(1), 76-88.

Nie, J. J., \& Deng, D. F. (2014). Manufacturer extended warranty services outsourcing strategy when product quality effect the cost of the extended warranty. Industrial Engineering and Management, 19(3), 26-32.

Wu P. (2019), Which battery-charging technology and insurance contract is preferred in the electric vehicle sharing business? Transportation Research Part A, 124 (2019), 537-548.

Schiffer, M., \& Walther, G. (2017). The electric location routing problem with time windows and partial recharging. European Journal of Operational Research, 260(8), 995-1013, doi: 10.1016/j.ejor.2017.01.011.

Šare, A., Krajačić1, G., Pukšec, T., and Duić, N..(2015) The integration of renewable energy sources and electric vehicles into the power system of the Dubrovnik region, Energy, Sustainability and Society, $5: 271-287$

Tong, P., Liu, Z., Men, F., \& Cao, L. (2014). Designing and pricing of two-dimensional extended warranty contracts based on usage rate. International Journal of Production Research, 52(21), 6362-6380.

Widrick, R. S., Nurre, S. G., \& Robbins, M. J. (2018). Optimal policies for the management of an electric vehicle battery swap station. Transportation Science, 52(1), 59-79.

Wu, H., Pang, G. K. H., Choy, K. L., \& Lam, H. Y. (2018). An Optimization Model for Electric Vehicle Battery Charging at a Battery Swapping Station. IEEE Trans, Veh. Technol., 67(2), 881-895.

Yao, C. Z., Luo, J. (2018). Deployment of a charging network based on a cost-benefit model and the CFSFDP algorithm. Kybernetes, K-08-2018-0420-. doi:10.1108/K-08-2018-0420.

Yang, J., \& Sun H. (2015). Battery swap station location-routing problem with capacitated electric vehicles. Computer Oper Res, 55(3), 217-232.

Yi, Y. Y., \& Yao, J. J. (2014). Supply chain coordination for extended warranty provided by strong retailer under network externality. Computer Integrated Manufacturing Systems, 20(8), 1987-1999.

Zhang, X., Zhang, B., \& Liang, D. (2016). Operation optimization of electricvehicles in battery swapping mode and direct charging mode in microgrid. Automat Electric Power Syst, 40(9), 56-63.

Zhao, D., Zhang, X. M., Ren, T. H., \& Fu, H. Y. (2019). Optimal pricing strategies in a product and service supply chain with extended warranty service competition considering retailer fairness concern. Mathematical Problems in Engineering, 8(27), 1-16.

Zheng, B., Bian, Y. W., Sun, Y. H., \& Ding, H. S. (2018). Optimal extended warranty strategy: Uniform or non-uniform pricing. International Transactions in Operational Research, doi:10.1111/itor.12611. 